\title{
Analysis of BRIP1 Variants among Korean Patients with BRCA1/2 Mutation-Negative High-Risk Breast Cancer
}

\section{Haeyoung Kim, $\mathrm{MD}^{1}$ \\ Dae-Yeon Cho, $\mathrm{PhD}^{2}$ \\ Doo Ho Choi, MD ${ }^{3}$ \\ Gee Hue Jung, MS² \\ Inkyung Shin, MS² \\ Won Park, MD ${ }^{3}$ \\ Seung Jae Huh, $\mathrm{MD}^{3}$ \\ Seok Jin Nam, MD \\ Jeong Eon Lee, $\mathrm{MD}^{4}$ \\ Won Ho Gil, MD ${ }^{4}$ \\ Seok Won Kim, MD 4}

${ }^{1}$ Department of Radiation Oncology, Hallym University Dongtan Sacred Heart Hospital, Hwaseong,

${ }^{2}$ LabGenomics Clinical Research Institute, LabGenomics, Seongnam,

Departments of ${ }^{3}$ Radiation Oncology and ${ }^{4}$ Surgery, Samsung Medical Center, Sungkyunkwan University School of Medicine, Seoul, Korea

\section{Purpose}

The aim of the current study is to assess the spectrum of genetic variation in the BRIP1 gene among Korean high-risk breast cancer patients who tested negative for the BRCA1/2 mutation.

\section{Materials and Methods}

Overall, 235 Korean patients with BRCA1/2 mutation-negative high-risk breast cancer were screened for BRIP1 mutations. The entire BRIP1 gene was analyzed using fluorescent-conformation sensitive gel electrophoresis. In silico analysis of BRIP1 variants was performed using PolyPhen-2 and SIFT.

\section{Results}

A total of 20 sequence alterations including 12 exonic and eight intronic variants were found. Among the 12 exonic variants, 10 were missense and two were silent mutations. No protein-truncating mutation was found among the tested patients. Among the 10 missense variants, four (p.L263F, p.L340F, p.L474P, and p.R848H) were predicted to be pathogenic by both PolyPhen-2 and SIFT, and these variants were found in five patients. Of the four missense variants, p.L263F, p.L474P, and p.R848H localize to regions between the helicase motifs, while p.L340F resides in an iron-sulfur domain of BRIP1.

\section{Conclusion}

No protein-truncating mutation in BRIP1 was found among the tested patients. The contribution of BRIP1 variants is thought to be minor in Korean non-BRCA1/2 high-risk breast cancer.

Correspondence: Doo Ho Choi, MD

Department of Radiation Oncology,

Samsung Medical Center,

Sungkyunkwan University School of Medicine,

81 Irwon-ro, Gangnam-gu, Seoul 06351, Korea

Tel: 82-2-3410-2436

Fax: 82-2-3410-2619

E-mail: doho.choi@samsung.com

Received June 3, 2015

Accepted December 25, 2015

Published Online January 19, 2016

*Haeyoung Kim and Dae-Yeon Cho contributed equally to this work.

Key words

BRIP1, BRCA1, BRCA2,

Hereditary breast and ovarian cancer syndrome, Korea

\section{Introduction}

Inherited mutations of certain genes contribute to the risk of breast cancer in approximately 15\% of breast cancer patients [1]. Among the susceptible genes for breast cancer, $B R C A 1$ and $B R C A 2$ (BRCA1/2) are considered most important as their mutations are associated with a high risk of breast cancer. Patients carrying deleterious mutations in one of the genes have a $40 \%-80 \%$ lifetime risk of breast cancer [2]. 
However, only about $20 \%$ of hereditary breast cancer is caused by the BRCA1/2 mutations, suggesting that other genes account for the remaining non-BRCA1/2-mutated hereditary breast cancer [2].

Korean patients with breast cancer are characteristically younger than their Caucasian counterparts. As of 2011, the median age of Korean breast cancer patients is 50 years. This is more than 10 years younger than that of patients in the United States [3]. Given a strong correlation between early occurrence of cancer and cancer susceptibility of patients, specific genetic alterations are believed to be linked to the development of breast cancer among Korean patients. Previous studies on Korean patients found that $8.6 \%-21.7 \%$ of patients with high-risk breast cancer have BRCA1/2 mutations [4]. In addition to $B R C A 1 / 2$, other genes including CHEK2 and PALB2 were tested for significance in predisposition to Korean breast cancer. No mutation was found in these genes among the tested patients $[5,6]$. Thus, a significant proportion of genetic causes of Korean high-risk breast cancer remain unknown.

BRCA1-interacting protein C-terminal helicase 1 (BRIP1) encodes a protein binding partner of $B R C A 1$ and functions in the process of DNA repair [7]. Sequence variants in BRIP1 are reported to be associated with development of breast cancer in Caucasian patients [2]. However, there is a paucity of data regarding the role of BRIP1 variants in Korean high-risk breast cancer. In the current study, we analyzed the BRIP1 gene in high-risk Korean breast cancer patients without the $B R C A 1 / 2$ mutation in order to assess the frequency of BRIP1 mutation among the patients.

\section{Materials and Methods}

\section{Patients and controls}

High-risk breast cancer patients without the BRCA1/2 mutation were enrolled in the current study. All participants were clinically screened for $B R C A 1 / 2$ mutations using fluorescent-conformation sensitive capillary electrophoresis (F-CSCE) and traditional sequencing. Detailed methods were previously described [8]. Because two previous studies reported that the large genomic rearrangements of $B R C A 1 / 2$ are rare in the Korean population [9], multiplex ligationdependent probe amplification analysis for large genomic rearrangement was not performed in the current study.

All patients were participants in the Korean Hereditary Breast Cancer (KOHBRA) study, conducted from 2008 to 2013 to evaluate the distribution of the BRCA1/2 mutation in Korean high-risk breast cancer patients [8]. Among the high- risk patients who did not have the $B R C A 1 / 2$ mutation, individuals who gave consent for analysis of the BRIP1 gene were included in the current research. The criteria for highrisk breast cancer patients were as follows: patients with a family history of breast or ovarian cancer in any relative; patients with breast cancer diagnosed at age 40 years or younger; patients with bilateral breast cancer; and male patients with breast cancer. In addition, 50 healthy females aged 28-36 years were selected as a control group for analysis of BRIP1 missense variants. The women in the control group underwent genetic testing during the prenatal screening program at the LabGenomics Clinical Research Institute and had no personal or familial history of breast or ovarian cancer. The current study was approved by the Institutional Review Board of the Ethics Committee of Samsung Medical Center.

\section{Mutation detection}

Genomic DNA was extracted from peripheral blood using a G-DEX Ilb DNA Extraction Kit (Intron, Seongnam, Korea), following the protocol of the manufacturer. Sequence variations in the BRIP1 gene were analyzed using the combination methods of F-CSCE and direct sequencing, based on the institute's own protocols, as described previously $[4,10]$. In short, fluorescent polymerase chain reaction (PCR) products were synthesized by amplifying 21 DNA fragments that overlapped the 19 coding exons and exon/intron boundaries of BRIP1. Primer sequences are available on request. Heteroduplexes were generated by denaturation and annealing of the PCR products, followed by separation on an $\mathrm{AB}$ 3130XL sequencer (Life Technology, Carlsbad, CA). F-CSCE peak pattern analysis was performed using GeneScane and Genotypere software (Life Technology). Following identification of a subset of PCR products with aberrant F-CSCE patterns, the PCR products were amplified using the same primers used for F-CSCE. These DNA fragments were sequenced in both directions on an AB 3730XL (Life Technology), in accordance with the manufacturer's instructions. All variants were confirmed by repeating both the PCR and sequencing analyses. All sequence variants are described according to the Human Genome Variation Society (HGVS) mutation nomenclature [11]. In the current study, variations are categorized as novel sequence variations when they cannot be found in publically available sequence variation databases including dbSNP (http://www.ncbi.nlm.nih.gov/snp/), Exome Aggregation Consortium (EXAC; http://exac.broadinstitute.org/), Leiden Open Variation Database (LOVD; http://www.lovd.nl/3.0/), Human Genome Mutation Database (HGMD; http://www.hgmd.cf.ac.uk/ac/), ClinVar (http:// www.ncbi.nlm.nih.gov/clinvar/), 1000 Genomes (http://www. 1000genomes.org/), and Exome Variant Server (http://evs. gs.washington.edu/EVS/). 
The in silico analyses of the BRIP1 variants were performed using Polymorphism Phenotyping ver. 2 (PolyPhen-2; http: //genetics.bwh.harvard.edu/pph2) and Sorting Intolerant From Tolerant (SIFT; http: //sift.jcvi.org/index.html) to predict the impact on the function of a protein. In the PolyPhen-2 program, the investigated mutation is categorized as probably damaging (probability score greater than 0.85 ), possibly damaging (probability score between 0.16 and 0.85 ), or benign (probability score less than or equal to 0.15 ). SIFT is a tool for sorting intolerant from tolerant amino acids in the base of a sequence homology. The evaluated amino acid substitution is predicted as deleterious if the score is less than 0.05 and is predicted to be tolerated if the score is greater than or equal to 0.05 . The missense variants predicted to be deleterious by in silico analyses were genotyped among females in the control group to compare frequencies of occurrence of the variants between the patients and the control groups.

\section{Results}

\section{Patient characteristics}

A total of 235 patients with breast cancer underwent genetic testing for BRIP1 mutations. Of the participants, 89 had familial breast cancer, 76 had early-onset breast cancer, 43 had early-onset breast cancer along with a family history of breast or ovarian cancer, six had bilateral breast cancers, eight had bilateral breast cancer along with a family history of breast or ovarian cancer, four had bilateral breast cancer and were diagnosed at age 40 years or younger, two had early-onset bilateral breast cancer along with a family history of breast or ovarian cancer, and seven were male patients with breast cancer. The median age at diagnosis was 46 years (range, 21 to 68 years).

\section{Genetic analysis of BRIP1}

Detailed data of BRIP1 sequence analysis are shown in Table 1. Overall, 20 BRIP1 sequence variants were found. Of the variants, 12 were located in protein-coding regions, and eight were in introns. Among the 12 exonic variants, there were 10 missense and two silent mutations. Notably, no protein truncating mutation was found among the tested patients. Among the 10 missense variants, two (c.787C $>$ T, and c.1421T $>C$ ) were novel and eight (c.430G $>A, c .587 A>G$, c. $1018 \mathrm{C}>$ T, с. $1442 \mathrm{G}>\mathrm{A}$, c. $2543 \mathrm{G}>\mathrm{A}$, c. $2755 \mathrm{~T}>\mathrm{C}, 2830 \mathrm{C}>\mathrm{G}$, and c. $2854 \mathrm{~A}>\mathrm{G}$ ) were previously described.

The effects of the 10 coding missense variants were ana- lyzed by PolyPhen-2 and SIFT. The c.787C >T (p.L263F), c.1018C > T (p.L340F), c.1421T>C (p.L474P), and c.2543G>A (p.R848H) variants were predicted to be pathogenic by both programs. These four missense mutations were found in five patients. Clinical information on the five patients is shown in Table 2. The four missense mutations were not detected among the females in the control group.

\section{Discussion}

In the current study, BRIP1 sequence variations including three novel variants were identified in Korean patients with non- $B R C A 1 / 2$ high-risk breast cancer. Among the variants, four missense variants were predicted to be deleterious by in silico analyses and were found to be rare, with a frequency of $1.27 \%$ in the study population. In addition, no proteintruncating BRIP1 mutation was identified among the tested patients. Therefore, it is less likely that variations in BRIP1 account for a significant proportion of non-BRCA1/2 highrisk breast cancer among Koreans.

BRIP1, also known as the BRCA1-associated C-terminal DNA helicase 1 (BACH1) or Fanconi anemia complementation group J (FANCJ), is a DNA helicase that directly interacts with the BRCT domain of BRCA1 and participates in DNA repair and checkpoint control [7]. Homozygous mutations in BRIP1 result in Fanconi anemia, which shows congenital anomalies, bone marrow failure, and increased prevalence of cancers [12]. In addition, inactivation of BRIP1 alters certain functions of $B R C A 1$, suggesting that $B R I P 1$ is a candidate breast cancer-associated gene [13]. Heterozygous germline mutations in BRIP1 are reported to increase the risk of breast cancer. A case-control study found that carriers of truncating mutations in BRIP1 showed a 2-fold increase in breast cancer risk compared to healthy individuals [12]. The study, which included 1,212 UK patients with non-BRCA1/2 high-risk breast cancer, found five distinctive truncating mutations among nine patients. In addition, three different truncating mutations have been identified in other studies; however, the frequencies of the protein-truncating variants was low at $0.7 \%-2.0 \%$ in the patients with non-BRCA1/2 highrisk breast cancer [14-16]. In addition, several studies conducted on patients with various ethnicities failed to identify BRIP1 protein-truncating variants in their study cohorts [1719]. Consistent with these studies, we found no BRIP1 protein-truncating variants in our study population, suggesting that the BRIP1 protein-truncating variant is less likely to contribute to the risk of breast cancer among Korean patients with non-BRCA1/2 high-risk breast cancer. Nonetheless, there is a possibility of missing BRIP1 truncating variants 


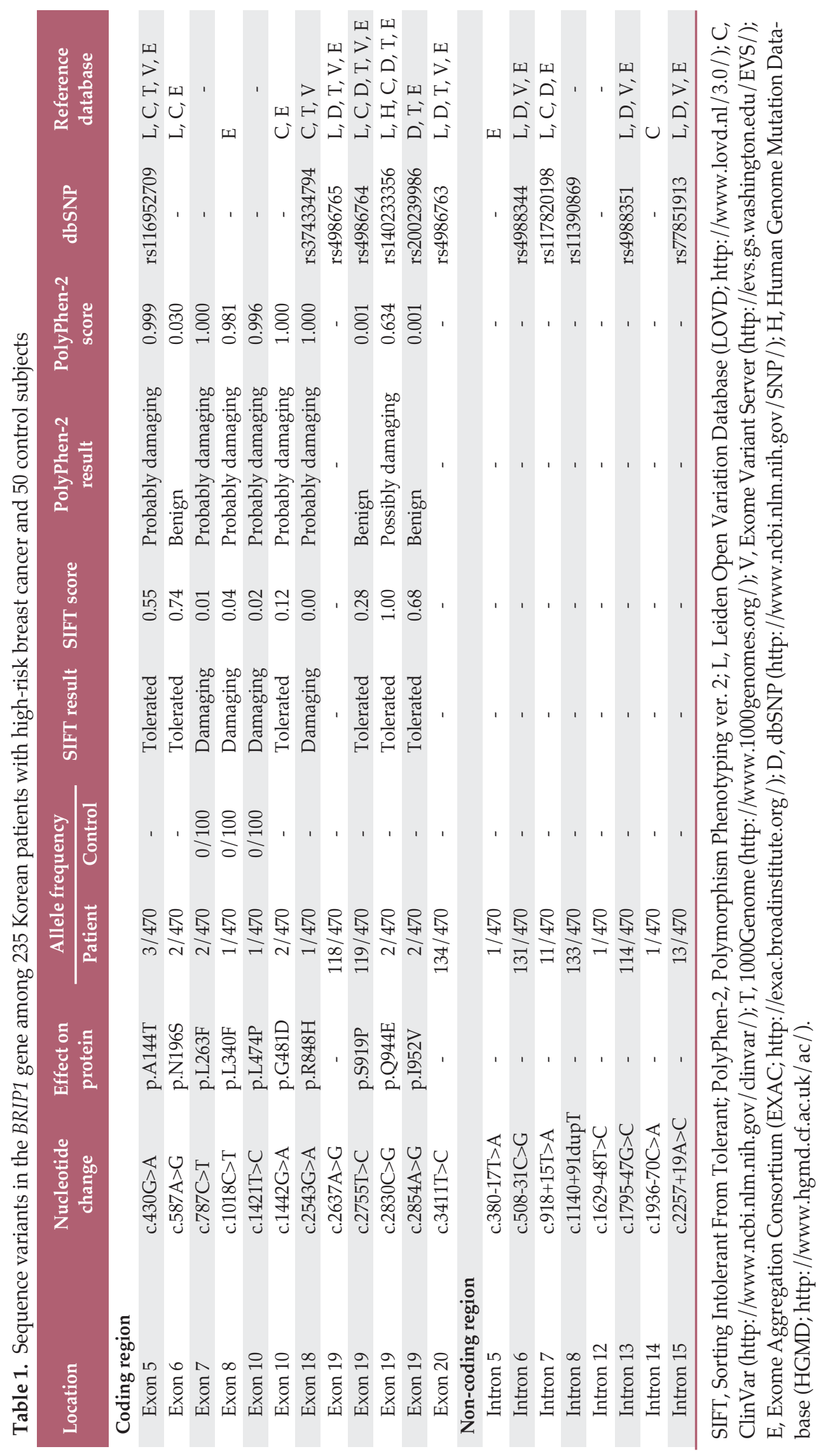




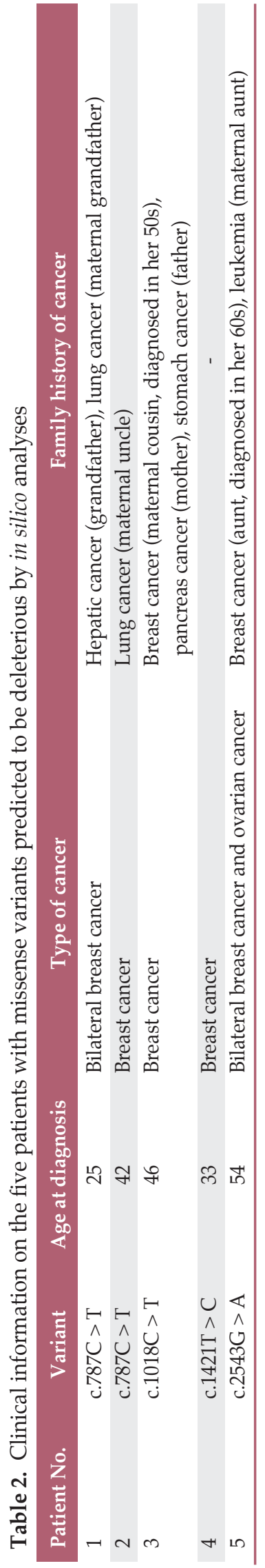

among Korean patients because we analyzed the BRIP1 sequence in only 235 patients with high-risk breast cancer. Further research including a larger number of patients is warranted to accurately estimate the frequency of BRIP1 truncating variants in Korean patients with breast cancer.

In addition to the protein-truncating BRIP1 mutations, missense variants in BRIP1 have been suggested to be related to breast cancer predisposition. Cantor and Andreassen [13] first described missense variants in BRIP1, p.P47A and p.M299I, among patients with early-onset breast cancer. Each variant was identified in one of the 86 patients with breast cancer, while the variants were not detected in healthy control subjects. In addition, the stability of BRIP1 containing the p.P47A mutant was markedly reduced in cultured cells [12]. However, p.P47A was confirmed to be unrelated to breast cancer predisposition in a case-control study including a large number of patients and controls [14]. Since these studies, several BRIP1 missense variants including p.R173C, p.V193I, p.Q944E, and p.P1034L have been identified and evaluated in terms of their roles in breast cancer susceptibility. Nonetheless, the contribution of BRIP1 missense variants to breast cancer predisposition remains controversial [20]. In the current study, we found 10 missense variants in the coding region of BRIP1, four of which were predicted to alter BRIP1 function by in silico analyses. Among the four missense variants, p.L263F, p.L474P, and p.R848H localize to regions between the helicase motifs of BRIP1. These variants are thought to be deleterious and are found among patients with high-risk breast cancer. However, functional evaluations of the variants and further case-control studies are needed to confirm their roles in breast cancer predisposition in Koreans.

Of note, we found a missense variant p.L340F, which resides in an iron-sulfur (Fe-S) domain of BRIP1 in one of the 235 patients in the current analysis. The patient had a family history of cancer on the maternal side, pancreas cancer in the mother, and breast cancer in a maternal cousin. The Fe-S domain of BRIP1 is proposed to play a fundamental role in DNA strand displacement, which is essential for helicase activity [21]. As a superfamily 2 helicase, BRIP1 contains seven conserved helicase motifs in residues 1-888, along with the Fe-S domain (residues 276-362) [7,21]. Previously, a missense mutation, p.A349P, which resides in the Fe-S domain of BRIP1, was found in a stillborn fetus who presented with a severe phenotypic abnormality and intrauterine growth failure [22]. A subsequent functional analysis of a recombinant BRIP1-A349P protein showed that the A349P mutant protein was unable to couple ATP hydrolysis and translocase activity, which is necessary for DNA unwinding [23]. Another missense variant, p.M299I, which localizes to the Fe-S domain, was identified in a case of early-onset breast cancer [7]. Unlike the A349 mutant, the recombinant BRIP1- 
M299I protein showed increased helicase activity compared to a wild-type recombinant BRIP1, along with proficient unwinding DNA substrates [24]. It is suggested that increased enzyme activity by p.M299I, which is caused by perturbation of the Fe-S cluster or structural effects, may contribute to cancer progression [25]. Based on the important functions of the Fe-S domain of BRIP1, the p.L340F variant found in the current study may have significant roles in breast cancer predisposition. The current study is the first to report a variant located in the Fe-S domain of BRIP1 since finding the p.M299I variant in patients with early-onset breast cancer. Functional effects of the sequence alteration should be evaluated in future studies.

Because protein-truncating mutation in BRIP1 was not identified in the current study, it is unlikely that alterations in BRIP1 significantly contribute to the susceptibility of breast cancer in Korean patients. Furthermore, even though BRIP1 missense variants were found, the role of the variants in the development of breast cancer among Korean patients with high-risk breast cancer is still inconclusive. Given the small sample size of the current study, there is a limitation in determining the precise function of BRIP1 mutation for breast cancer occurrence in Koreans. Further research including a large number of patients and control subjects is necessary.

\section{Conclusion}

Several sequence alterations in BRIP1 were found among the tested patients, suggesting that BRIP1 sequence alterations have effects on breast predisposition among Korean breast cancer patients. However, given that no protein-truncating mutation was found and that the frequency of the sequence variations was low, the contribution of BRIP1 variants is thought to be minor in Korean non-BRCA1/2 high-risk breast cancer.

\section{Conflicts of Interest}

Conflict of interest relevant to this article was not reported.

\section{Acknowledgments}

This study was mainly supported by a grant from the Samsung Biomedical Research Institute (\#SBRI C-B1-132-1) and was supported in part by the Korea Health Technology R\&D Project through KHIDI, funded by the Ministry of Health \& Welfare (\#HI13C2096), Republic of Korea.

\section{References}

1. Daly MB, Axilbund JE, Buys S, Crawford B, Farrell CD, Friedman S, et al. Genetic/familial high-risk assessment: breast and ovarian. J Natl Compr Canc Netw. 2010;8:562-94.

2. Hollestelle A, Wasielewski M, Martens JW, Schutte M. Discovering moderate-risk breast cancer susceptibility genes. Curr Opin Genet Dev. 2010;20:268-76.

3. Kim Z, Min SY, Yoon CS, Lee HJ, Lee JS, Youn HJ, et al. The basic facts of korean breast cancer in 2011: results of a nationwide survey and breast cancer registry database. J Breast Cancer. 2014;17:99-106.

4. Kim H, Cho DY, Choi DH, Choi SY, Shin I, Park W, et al. Characteristics and spectrum of BRCA1 and BRCA2 mutations in 3,922 Korean patients with breast and ovarian cancer. Breast Cancer Res Treat. 2012;134:1315-26.

5. Choi DH, Cho DY, Lee MH, Park HS, Ahn SH, Son BH, et al. The CHEK2 1100delC mutation is not present in Korean patients with breast cancer cases tested for BRCA1 and BRCA2 mutation. Breast Cancer Res Treat. 2008;112:569-73.

6. Kim JH, Choi DH, Cho DY, Ahn SH, Son BH, Haffty BG.
PALB2 mutations 1592delT and 229delT are not present in Korean breast cancer patients negative for BRCA1 and BRCA2 mutations. Breast Cancer Res Treat. 2010;122:303-6.

7. Cantor SB, Bell DW, Ganesan S, Kass EM, Drapkin R, Grossman S, et al. BACH1, a novel helicase-like protein, interacts directly with BRCA1 and contributes to its DNA repair function. Cell. 2001;105:149-60.

8. Han SA, Park SK, Ahn SH, Lee MH, Noh DY, Kim LS, et al. The Korean Hereditary Breast Cancer (KOHBRA) study: protocols and interim report. Clin Oncol (R Coll Radiol). 2011; 23:434-41.

9. Seong MW, Cho SI, Noh DY, Han W, Kim SW, Park CM, et al. Low contribution of BRCA1/2 genomic rearrangement to high-risk breast cancer in the Korean population. Fam Cancer. 2009;8:505-8

10. Ahn SH, Son BH, Yoon KS, Noh DY, Han W, Kim SW, et al. BRCA1 and BRCA2 germline mutations in Korean breast cancer patients at high risk of carrying mutations. Cancer Lett. 2007;245:90-5. 
11. den Dunnen JT, Antonarakis SE. Mutation nomenclature extensions and suggestions to describe complex mutations: a discussion. Hum Mutat. 2000;15:7-12.

12. Ameziane N, van den Ouweland AM, Adank MA, Vijzelaar RN, Errami A, Dorsman JC, et al. Lack of large genomic deletions in BRIP1, PALB2, and FANCD2 genes in BRCA1/2 negative familial breast cancer. Breast Cancer Res Treat. 2009;118: 651-3.

13. Cantor SB, Andreassen PR. Assessing the link between BACH1 and BRCA1 in the FA pathway. Cell Cycle. 2006;5: 164-7.

14. Seal S, Thompson D, Renwick A, Elliott A, Kelly P, Barfoot R, et al. Truncating mutations in the Fanconi anemia J gene BRIP1 are low-penetrance breast cancer susceptibility alleles. Nat Genet. 2006;38:1239-41.

15. Wong MW, Nordfors C, Mossman D, Pecenpetelovska G, Avery-Kiejda KA, Talseth-Palmer B, et al. BRIP1, PALB2, and RAD51C mutation analysis reveals their relative importance as genetic susceptibility factors for breast cancer. Breast Cancer Res Treat. 2011;127:853-9.

16. De Nicolo A, Tancredi M, Lombardi G, Flemma CC, Barbuti $S$, Di Cristofano C, et al. A novel breast cancer-associated BRIP1 (FANCJ/BACH1) germ-line mutation impairs protein stability and function. Clin Cancer Res. 2008;14:4672-80.

17. Kuusisto KM, Bebel A, Vihinen M, Schleutker J, Sallinen SL. Screening for BRCA1, BRCA2, CHEK2, PALB2, BRIP1, RAD50, and CDH1 mutations in high-risk Finnish BRCA1/ 2-founder mutation-negative breast and/or ovarian cancer individuals. Breast Cancer Res. 2011;13:R20.
18. Cao AY, Huang J, Hu Z, Li WF, Ma ZL, Tang LL, et al. Mutation analysis of BRIP1/BACH1 in BRCA1/BRCA2 negative Chinese women with early onset breast cancer or affected relatives. Breast Cancer Res Treat. 2009;115:51-5.

19. Catucci I, Milgrom R, Kushnir A, Laitman Y, Paluch-Shimon S, Volorio S, et al. Germline mutations in BRIP1 and PALB2 in Jewish high cancer risk families. Fam Cancer. 2012;11:483-91.

20. Cantor SB, Guillemette S. Hereditary breast cancer and the BRCA1-associated FANCJ/BACH1/BRIP1. Future Oncol. 2011;7:253-61.

21. Rudolf J, Makrantoni V, Ingledew WJ, Stark MJ, White MF. The DNA repair helicases XPD and FancJ have essential ironsulfur domains. Mol Cell. 2006;23:801-8.

22. Levran O, Attwooll C, Henry RT, Milton KL, Neveling K, Rio $\mathrm{P}$, et al. The BRCA1-interacting helicase BRIP1 is deficient in Fanconi anemia. Nat Genet. 2005;37:931-3.

23. Wu Y, Sommers JA, Suhasini AN, Leonard T, Deakyne JS, Mazin AV, et al. Fanconi anemia group J mutation abolishes its DNA repair function by uncoupling DNA translocation from helicase activity or disruption of protein-DNA complexes. Blood. 2010;116:3780-91.

24. Gupta R, Sharma S, Doherty KM, Sommers JA, Cantor SB, Brosh RM Jr. Inhibition of BACH1 (FANCJ) helicase by backbone discontinuity is overcome by increased motor ATPase or length of loading strand. Nucleic Acids Res. 2006;34:6673-83.

25. Wu Y, Brosh RM Jr. DNA helicase and helicase-nuclease enzymes with a conserved iron-sulfur cluster. Nucleic Acids Res. 2012;40:4247-60. 